\title{
Mediterranean Diet Trial on Health, Longevity and Prevention of Chronic Cardiovascular Diseases: A Preliminary Announcement
}

\author{
N Angelides*, B Balas, S Novo, J Fernandes, L Antignani, E Hussein, Ch Liapis, P Poredos and E \\ Andreou \\ Cardiovascular and Thoracic Unit, Nicosia General Hospital, Nicosia, Cyprus
}

*Corresponding author: N Angelides, Cardiovascular and Thoracic Unit, Nicosia General Hospital, Nicosia, Cyprus

\begin{tabular}{lll}
\hline ARTICLE INFO & & ABSTRACT \\
\cline { 1 - 1 } Received: $\begin{array}{ll}\text { Citation: N Angelides, B Balas, S Novo, J Fernandes, L Antignani, et al., Mediterranean } \\
\text { Published: } 1521\end{array}$ & $\begin{array}{l}\text { Diet Trial on Health, Longevity and Prevention of Chronic Cardiovascular Diseases: A } \\
\text { Preliminary Announcement. Biomed J Sci \& Tech Res 36(4)-2021. BJSTR. MS.ID.005899. }\end{array}$ \\
\hline
\end{tabular}

\section{Opinion}

The Mediterranean Diet (Med-D) can be defined as a healthy eating pattern contributing to overall health and longevity. According to the individual heritage and cultures of local populations, Med-D, integrates a number of variations on a basic diet which is common in all the Mediterranean Countries. Med-D, includes plant foods such as fruits, vegetables, cereals, nuts, seeds, and lots of olive oil. Also, dairy products with lower consumption of milk and higher consumption of low-fat cheese, yoghurt and fish, moderate consumption of eggs and chickens, very low amounts of red meat and low to moderate consumption of wine, mainly with evening meals. Med-D, favors local and seasonal food products to a much greater degree. In 2016, a decision was taken by the Executive Board of the Mediterranean League of Angiology and Vascular Surgery to monitor an open study on Med-D, in order to analyze the effects of this diet based on a protocol and a well-balanced questionnaire of 21 pages; this study has started to enroll subjects in January 2017 taking into account the MED-D Score described by UNESCO, and other validated guidelines.

In 2018, a pilot study was presented at the MLAVS Congress in Palermo on 138 volunteers with a range of age between 20 and 80 years using the MLAVS questionnaire in order to see its flexibility in practice. A second preliminary attempt on Med-D study, carried out using the same protocol in 6 Mediterranean Countries (Cyprus, Egypt, Greece, Italy, France and Slovenia) based on 500 volunteers
(Cyprus $n=150$, Egypt $n=50$, Italy $n=200$, France $n=50$, Slovenia $\mathrm{n}=50$ ). The percentage of men was 51.5 and of women 48.5 . The questionnaire, included questions concerning the medical history of the subjects. In the specific question of whether volunteers had suffered from health problems of chronic nature, 35.2\% answered positively and $64.8 \%$ negatively. Also, $35.3 \%$ were receiving some type of medication at the time of the study and $64.7 \%$ were not. Only $14.4 \%$ followed a diet prescribed by a dietitian, whereas $85.6 \%$ did not follow a specific diet. From all the subjects, $35.3 \%$ were taking nutritional supplements and $64.7 \%$ were not.

Dyslipidemia, had the highest percentage (22.4\%) from all the health conditions. This, was followed by lumbago $(13,4 \%)$, hypertension (11.3\%), menopause (9,9\%), gastritis/gastric ulcer (6.5\%), and osteoporosis/osteopenia (5.8\%). A major part of the questionnaire, investigated the nutritional habits of the adult population. In the specific question if breakfast and/or a midmorning snack was consumed, $24.2 \%$ of the subjects reported that they took breakfast only, $11.1 \%$ mid-morning snack only, $61 \%$ had both and $3.7 \%$ had neither breakfast nor a mid-morning snack. The choices for breakfast were as follows: $84.3 \%$ of the sample consumed milk with sugar free cereals and olives, 51.7\% milk with bread, $63.8 \%$ milk with bread and marmalade, $76.0 \%$ toasts with olive oil and $19.0 \%$ humus. The most popular foods for midmorning snack was $79.7 \%$ for fresh fruit, $54.7 \%$ for bakery goods, $61.2 \%$ for sandwiches, and, $68.8 \%$ for coffee only. The meals eaten 
per day, were $2.3+/-0.9$ and the number of snacks per day were $1.9+/-1.12$.

According to our study $76.8 \%$ of the participants eat breakfast regularly, $56.9 \%$ eat lunch, $60.2 \%$ eat dinner and $39.9 \%$ eat intermediate meals. In our study, the use of olive oil in green salads was $98.3 \%$ and for cooking purposes was $62.8 \%$. The study showed that $29.0 \%$ used another type of vegetable oil, and $2.6 \%$ did not know what oil they use; $3.1 \%$ did not cook at home, and $0.4 \%$ use butter. The adults' majority drink either 1-4 glasses of water (35.2\%); 5- 8 glasses of water $(37.3 \%)$ and $25.3 \%$ drink more than 8 glasses of water. The large variety of answers with regards to the consumption of water was related to the seasonal changes of temperature. Our study, also investigated the level of physical activity in the population. On an average day, the subjects spend $9.8+/-5.0$ hours sitting. On the question of how physically demanding their job was, $54.1 \%$ reported very little, $31.6 \%$ just little, $7.7 \%$ fairly demanding, and $6.6 \%$ heavily demanding. During a typical week, the subjects spent $0.43+/-1.21$ days engaged in intense physical activity, 1.1+/- 2.08 days engaged in medium intensity physical activity, and $1.6+/-2.28$ days engaged in lowlevel intensity physical activity.

At this stage of the study and taking into consideration the number of volunteers and their different Mediterranean origin, we would like to present preliminary but valid results on the preventing effect of Med-D, concerning certain chronic diseases. Doubtful results were not included. We found that Med-D protects against type 2 diabetes. Emphasis is given on rich in mono-unsaturated fats and fibers foods, like fruits and vegetables, fish and olive oil. These ingredients, seems to make the Mediterranean diet capable to lower blood sugar in diabetics. Med-D, maintains the levels of cholesterol in the blood. It reduces the risk of cardiovascular mortality, due to its positive impact on HD cholesterol that can accumulate in deposits in the arteries. Similarly, Med-D, keeps people fit as they getting older. Thanks to all the nutrients, vitamins, and minerals that are consumed with the Mediterranean diet, there is a reduced risk of developing early muscle weakness and other aging problems, provided that a reasonable exercise is taking place too.

Med-D also reduces the risk of developing Alzheimer's and other mental diseases. For seniors, the Mediterranean diet can protect against mental decline enabling aging adults to preserve their quality of life. Similarly, the antioxidants, which are found in Med-D, keep cells from undergoing the process of oxidative stress, which causes damage to the body and can contribute to the development of degenerative diseases, like Parkinson's. It also helps people to lose weight, as it is easier to stick on this diet for a long- term period or even through life. It has been shown that Med-D diet can lead to a reduced risk of developing cancer. It seems that there is a protective action of this diet when it comes to cancer prevention. In particular, it helps to prevent the development of post-menopausal breast cancer. However, we cannot absolutely confirm these findings, so far. Finally, Med-D, fights against inflammation. Since a major trigger for inflammation is the exposure to oxidative stress, it follows that the high concentration of antioxidants in Med-D, can boost the defense system of the body. Mortality statistics from the Mediterranean Countries during the last 50 years, confirmed previous studies which have shown a stable beneficial effect of Med-D on health and longevity. We hope that in the future MLAVS Med-D Study will shed more light and evidence on this crucial matter.
ISSN: 2574-1241

DOI: 10.26717/BJSTR.2021.36.005899

N Angelides. Biomed J Sci \& Tech Res

CC This work is licensed under Creative Commons Attribution 4.0 License

Submission Link: https://biomedres.us/submit-manuscript.php

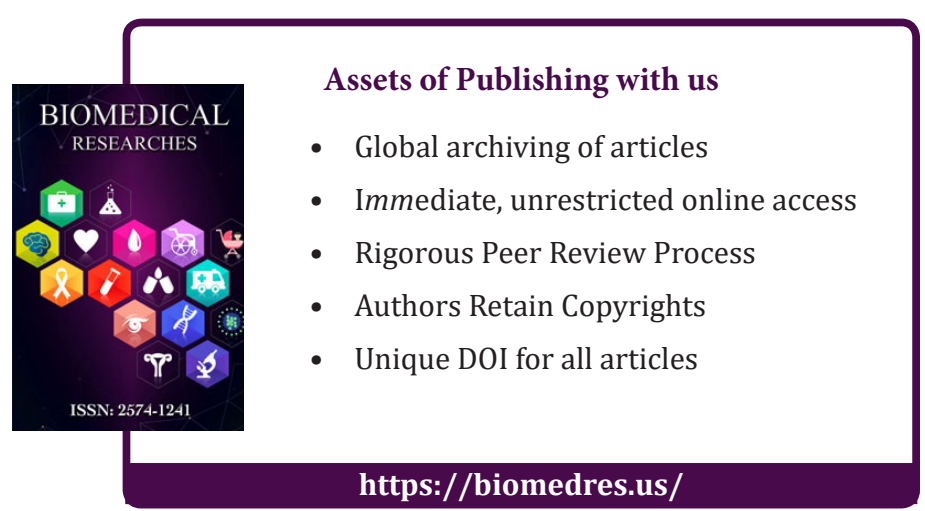

